\title{
Study on Identification of Pasturage Sources of Stingless Bee (Trigona iridipennis Smith.)
}

\author{
A.N. Roopa ${ }^{1}$, G. Eswarappa ${ }^{2}$, Sanganna M. Sajjanar ${ }^{3 *}$ and Govi Gowda ${ }^{2}$ \\ ${ }^{1}$ Deputy Director of Agriculture, Directorate office, Bengaluru, Karnataka, India \\ ${ }^{2}$ Department of Apiculture, UAS, GKVK, Bengaluru, Karnataka, India \\ ${ }^{3}$ Farm Superintendent, Agriculture Research Station, Siruguppa (UASR), Karnataka, India \\ *Corresponding author
}

\section{A B S T R A C T}

\section{Keywords}

Pasturage sources, Stingless bee.

Article Info

Accepted:

10 September 2017

Available Online:

10 November 2017
Total of 107 plant species belong to 45 families were found to yield pollen or nectar or both to the T. iridipennis. 13 field crops comprising 6 family, 2 spices and condiments (2-family), 10 vegetable crops (4-family), 14 fruits and plantation crops (13-family), 12 ornamental plants (10-family), 19 medicinal plants (11-family), 10 weeds (7-family) and 27 trees (14-family). Worker bees of $T$. iridipennis were collected resin from four plants viz., jack fruit, drumstick, mango and perivinkle at Bangalore region.

\section{Introduction}

Stingless bees are social insects that have existed for over 100 million years, nesting in dark places consist of hundreds to thousands of individuals, both adults and brood, the responsibility of bringing food depends on small group of workers (Wille, 1983). They can be domesticated by transferring natural colonies to manmade bee hives for production of honey, pollen, resin and pollination of crops. The stingless bees are generalist flower visitors and their foraging range is very shorter than true honeybees. The frequency of bee visitation is more on plants which are very nearer to the colony. The information on foraging for the pollen, nectar or collection of both from various flowering plants is very much limited. Hence, study on identification of different pasturage source of stingless bees has carried out at Regional Research Station, UAS, GKVK, Bangaluru.

\section{Materials and Methods}

In situ observations were made while stingless bees were foraging on plants to find out pasturage sources that provided nectar and pollen. The sources that provide resin to stingless bees were also recorded. The plant was judged as nectar source when bees inserted proboscis into flower for sipping nectar and as pollen source when corbiculae got loaded with pollen during foraging. When both the activities observed on the same plant was considered as a source for both nectar 
and pollen. Similarly, the plant was regarded as a resin source when the corbiculae of stingless bees got loaded with sticky material.

\section{Results and Discussion}

Trigona iridipennis was found to forage on 107 plant species belonging to 45 families, of which 62 plants provided pollen, 8 plants offered nectar and 37 plants offered both. Among 107 plants, 13 field crops, 2 spices and condiments, 10 vegetables crops, 14 fruit and plantations crops, 12 ornamental plants, 19 medicinal plants, 10 weeds and 27 trees (Table 1). The family wise distribution of pasturage sources is presented in Table 2. Among forage plants of Trigona iridipennis, the members of the family Fabaceae topped the list followed by Asteraceae and Cucurbitaceae. The members of these families being highly cross pollinated, they seem to have co-evolved with the bees by providing nectar and pollen and in turn getting pollinated by them. Species of genus Scaptotrigona showed special preference to eucalyptus flowers for collection of both pollen and nectar even though they visited hundred plants (Ramalho et al., 1980). Trigona iridipennis attracted to both minute flowers (ex. Bidens pilosa) and large showy flower (ex. Delonix regia). Few generalization could be made regarding flower preferred by stingless bees viz. Vitex spp., Lawsonia inermis flowers were very attractive to these bee as they preferred small flowers, dense inflorescence with corolla tube shorter than their tongue length and flowers with white corolla tube permitting their entry inside ex. Sesamum indicum (Heard, 1999). Trigona iridipennis preferred by white and yellow flowers like copper pod, mango, etc., rather than pink flowers.

Table.1 Pasturage sources of Trigona iridipennis at GKVK, Bangalore

\begin{tabular}{|c|c|c|c|c|c|c|}
\hline $\begin{array}{l}\text { Sl. } \\
\text { No. }\end{array}$ & $\begin{array}{c}\text { Common } \\
\text { name }\end{array}$ & Scientific name & Local name & Family & Source & $\begin{array}{l}\text { Method of } \\
\text { evaluation }\end{array}$ \\
\hline \multicolumn{7}{|c|}{ Field crops } \\
\hline \multicolumn{7}{|c|}{ Cereals } \\
\hline 01 & Finger millet & Eleusine coracana Gaertn. & Ragi & Poaceae & $\mathrm{P}$ & MA \\
\hline 02 & Pearl millet & $\begin{array}{l}\text { Pennisetum typhoides } \\
\text { Stapt an Hubb. }\end{array}$ & Sajje & Poaceae & $\mathrm{P}$ & VO \\
\hline 03 & Maize & Zea mays L. & $\begin{array}{l}\text { Musukina jola } \\
\text { (Mekkejola) }\end{array}$ & Poaceae & $\mathrm{P}$ & $\mathrm{VO}$ \\
\hline 04 & Sorghum & $\begin{array}{l}\text { Sorghum bicolor Linn. } \\
\text { Mocch. }\end{array}$ & Jola & Poaceae & $\mathrm{P}$ & $\mathrm{VO}$ \\
\hline \multicolumn{7}{|c|}{ b. $\quad$ Pulses } \\
\hline 01 & Cowpea & Vigna catjung Walp. & $\begin{array}{l}\text { Alasandhi } \\
\text { (Thadagani) }\end{array}$ & Fabaceae & $\mathrm{N}$ & $\mathrm{VO}$ \\
\hline 02 & Blackgram & Phaseolus mungo L. & Uddhu & Fabaceae & $\mathrm{N}$ & $\mathrm{VO}$ \\
\hline 03 & Greengram & Vigna radiate var. Nilezek & Hesaru & Fabaceae & $\mathrm{N}$ & $\mathrm{VO}$ \\
\hline 04 & Redgram & Cajanus cajan L. & Thogari & Fabaceae & NP & $\mathrm{VO}$ \\
\hline \multicolumn{7}{|c|}{ Oil seeds } \\
\hline 01 & Sunflower & Helianthus annuиs L. & Suryakanti & Compositae & NP & $\mathrm{VO}$ \\
\hline 02 & Niger & Guizotia abyssinica Cass. & Huchchellu & Compositae & NP & $\mathrm{VO}$ \\
\hline 03 & Seasamum & Sesamum indicum L. & Ellu & Pedaliaceae & $\mathrm{N}$ & $\mathrm{VO} / \mathrm{MO}$ \\
\hline 04 & Mustard & Brassica juncea Corr. & Sasive & Brassicaceae & NP & $\mathrm{VO}$ \\
\hline 05 & Castor & Ricinus communis L. & Haralu & Euphorbiaceae & $\mathrm{P}$ & $\mathrm{VO}$ \\
\hline
\end{tabular}




\begin{tabular}{|c|c|c|c|c|c|c|}
\hline 01 & Coriander & Coriandrum sativum $\mathrm{L}$. & Dhaniya & Umbelliferae & NP & $\mathrm{VO}$ \\
\hline 02 & Curry leaves & Murraya koenigii Spreng. & Karibevu & Rutaceae & $\mathrm{P}$ & $\mathrm{VO}$ \\
\hline \multicolumn{7}{|c|}{ III. Vegetables } \\
\hline 01 & Drumstick & Moringa oleifera Lamk. & Nugge & Moringaceae & $\mathrm{P}$ & $\mathrm{VO} / \mathrm{MA}$ \\
\hline 02 & Onion & Allium cepa $\mathrm{L}$. & Irulli & Liliaceae & NP & VO \\
\hline 03 & Brinjal & Solanum melongena L. & Badane & Solanaceae & $\mathrm{P}$ & VO/MA \\
\hline 04 & Chow-chow & $\begin{array}{l}\text { Sechium edule (Jacq.) } \\
\text { Swartz }\end{array}$ & Semebadane & Cucurbitaceae & NP & VO \\
\hline 05 & Pumpkin & Cucurbita pepo Dc. & Kumbalakai & Cucurbitaceae & $\mathrm{P}$ & $\mathrm{VO}$ \\
\hline 06 & Cucumber & Cucumis sativus L. & Sauthekai & Cucurbitaceae & $\mathrm{P}$ & $\mathrm{VO}$ \\
\hline 07 & Ridgegourd & Luffa acutangula $\mathrm{L}$. & Heerekai & Cucurbitaceae & $\mathrm{P}$ & $\mathrm{VO}$ \\
\hline 08 & Bittergourd & Momordica charantia $\mathrm{L}$. & Hagalakai & Cucurbitaceae & $\mathrm{P}$ & $\mathrm{VO}$ \\
\hline 09 & Bottlegourd & Lagenaria vulgaris $\mathrm{Sr}$. & Sorekai & Cucurbitaceae & $\mathrm{P}$ & $\mathrm{VO}$ \\
\hline 10 & Ashgourd & Benincasa hispida Cogn. & Budugumbala & Cucurbitaceae & $\mathrm{P}$ & $\mathrm{VO}$ \\
\hline \multicolumn{7}{|c|}{ IV. $\quad$ Fruits and plantations } \\
\hline 01 & Mango & Mangifera indica $\mathrm{L}$. & Mavu & Anacardiaceae & NP & VO/MA \\
\hline 02 & Papaya & Carica papaya $\mathrm{L}$. & Parangi & Caricaceae & $\mathrm{P}$ & $\mathrm{VO}$ \\
\hline 03 & Grapes & Vitis vinifera $\mathrm{L}$. & Drakshi & Vitaceae & $\mathrm{P}$ & $\mathrm{VO}$ \\
\hline 04 & Pomogranate & Punica granatum $\mathrm{L}$. & Dalimbe & Punicaceae & $\mathrm{P}$ & VO \\
\hline 05 & Simarouba & Simarouba glauca & Swargadhamara & Simaraubaceae & NP & $\mathrm{VO}$ \\
\hline 06 & Jack & Artocarpus integrifolia L. & Halasinamara & Moraceae & $\mathrm{P}$ & $\mathrm{VO}$ \\
\hline 07 & Banana & Musa paradisiacal L. & Bale & Moraceae & $\mathrm{N}$ & $\mathrm{VO}$ \\
\hline 08 & Arecanut & Areca catechu & Adike & Arecaceae & NP & $\mathrm{VO}$ \\
\hline 09 & Coconut & Cocos nucifera $\mathrm{L}$. & Thengu & Arecaceae & NP & $\mathrm{VO}$ \\
\hline 10 & Guava & Psidium guazava $\mathrm{L}$. & Sebekai & Myrtaceae & $\mathrm{P}$ & $\mathrm{VO}$ \\
\hline 11 & Gooseberry & Phyllanthus emblica & Kiruneeli & Euphorbiaceae & NP & VO \\
\hline 12 & Acid lime & Citrus meica var. acida & Nimbe & Rutaceae & NP & $\mathrm{VO}$ \\
\hline 13 & $\begin{array}{l}\text { Singapur } \\
\text { cherry }\end{array}$ & Muntingia calabora & Gasagase mara & Eleocarpaceae & NP & VO/MA \\
\hline 14 & Tamarind & Tamarindus indica D.C. & Hunase & Caesalpinaceae & NP & VO/MA \\
\hline \multicolumn{7}{|c|}{$\begin{array}{ll}\text { V. } & \text { Ornamentals } \\
\end{array}$} \\
\hline 01 & Tuberose & Palianthus tuberose Linn. & Sugandaaraju & Agaraceae & NP & $\mathrm{VO}$ \\
\hline 02 & Bottlebrush & $\begin{array}{l}\text { Callestemon lanceolus } \\
\text { D.C. }\end{array}$ & Bottlebrush & Myrtaceae & NP & $\mathrm{VO}$ \\
\hline 03 & $\begin{array}{l}\text { Railway } \\
\text { creeper }\end{array}$ & Ipomea cairica & Bekkenahejje & Convolvulaceae & NP & $\mathrm{VO}$ \\
\hline 04 & $\begin{array}{l}\text { Rangoon } \\
\text { creeper }\end{array}$ & Quisqualis indica $\mathrm{L}$. & Beeplant & Combretaceae & $\mathrm{N}$ & $\mathrm{VO}$ \\
\hline 05 & Berleria & Berleria cristata $\mathrm{L}$. & Spatikadahoo & Acanthaceae & NP & VO/MA \\
\hline 06 & Hibiscus & Hibiscus rosa sinensis & Dhasavala & Malvaceae & $\mathrm{P}$ & $\mathrm{VO}$ \\
\hline 07 & Rose & Rosa indica $\mathrm{L}$ & Gulabi & Rosaceae & $\mathrm{P}$ & $\mathrm{VO}$ \\
\hline 08 & Salvia & Salvia splendenus & Salvia & Lamiaceae & $\mathrm{P}$ & $\mathrm{VO}$ \\
\hline 09 & $\begin{array}{l}\text { Garden } \\
\text { balsam }\end{array}$ & Impatiens balsamina $\mathrm{L}$. & Karnakundala & Balsaminae & $\mathrm{P}$ & $\mathrm{VO}$ \\
\hline 10 & Marigold & Aster sp. & Chenduhoo & Asteraceae & $\mathrm{P}$ & $\mathrm{VO}$ \\
\hline 11 & Cosmos & Cosmos suphureas Cav. & Cosmos & Asteraceae & $\mathrm{P}$ & $\mathrm{VO}$ \\
\hline 12 & Golden rod & Salidago sp. & Goldenrod & Asteraceae & $\mathrm{P}$ & $\mathrm{VO}$ \\
\hline \multicolumn{7}{|c|}{ VI. $\quad$ Medicinal plants } \\
\hline 01 & Honey plant & Ammi majus & Madhugida & Umbelliferae & NP & $\mathrm{VO}$ \\
\hline
\end{tabular}




\begin{tabular}{|c|c|c|c|c|c|c|}
\hline 02 & $\begin{array}{l}\text { Bishops } \\
\text { weed }\end{array}$ & Ammi vasnaga & - & Umbelliferae & NP & $\mathrm{VO}$ \\
\hline 03 & Ajwan & Trachysprmum amni & Omum & Umbelliferae & $\mathrm{P}$ & $\mathrm{VO}$ \\
\hline 04 & $\begin{array}{l}\text { Indian } \\
\text { acalypha }\end{array}$ & Acalypha indica & Acalypha & Euphorbiaceae & NP & $\mathrm{VO}$ \\
\hline 05 & Physic nut & Jatropha curcas & Ritha & Euphorbiaceae & $\mathrm{P}$ & $\mathrm{VO}$ \\
\hline 06 & Kalama dye & Mallotus philippensis & Kunkuma & Euphorbiaceae & $\mathrm{P}$ & $\mathrm{VO}$ \\
\hline 07 & $\begin{array}{l}\text { Indian } \\
\text { liquorice }\end{array}$ & Arbus precororius & Jestiamadhu & Fabaceae & $\mathrm{P}$ & $\mathrm{VO}$ \\
\hline 08 & Babchi & Psoralea corylifolia & Baranchi gida & Fabaceae & $\mathrm{P}$ & $\mathrm{VO}$ \\
\hline 09 & Neeligida & Indigofera tinctoria & Neeligida & Fabaceae & $\mathrm{P}$ & $\mathrm{VO}$ \\
\hline 10 & Alfalfa & Medicago sativa & Kudarer masale & Fabaceae & $\mathrm{P}$ & VO \\
\hline 11 & Tube flower & Clerodendrum serratum & Tube flower & Verbanaceae & $\mathrm{P}$ & $\mathrm{VO}$ \\
\hline 12 & Dioscorea & Dioscorea composita & Dioscorea gadde & Diascoreaceae & $\mathrm{P}$ & $\mathrm{VO}$ \\
\hline 13 & Prim rose & Ocnothera lamarkiana & Kadu garagalu & Onagrceae & $\mathrm{P}$ & $\mathrm{VO}$ \\
\hline 14 & $\begin{array}{l}\text { Sweet worn } \\
\text { wood }\end{array}$ & Artemisia annиa & Artemisia & Asteraceae & $\mathrm{P}$ & $\mathrm{VO}$ \\
\hline 15 & Sylibium & Sylibum marianmum & Holithesal & Asteraceae & $\mathrm{P}$ & $\mathrm{VO}$ \\
\hline 16 & Rauvolfia & Rauvolfia tetraphylla & Sarpagandha & Apocyanaceae & $\mathrm{P}$ & $\mathrm{VO}$ \\
\hline 17 & Ocimum & Ocimum sanctum & Tulasi & Lamiaceae & NP & $\mathrm{VO}$ \\
\hline 18 & Lea & Lea indica & Andilu & Vitaceae & $\mathrm{P}$ & $\mathrm{VO}$ \\
\hline 19 & Garden & Ruta graveolens & Nagadali & Rutaceae & $\mathrm{P}$ & $\mathrm{VO}$ \\
\hline \multicolumn{7}{|c|}{ VII. Weeds } \\
\hline 01 & $\begin{array}{l}\text { Tenners } \\
\text { cassia }\end{array}$ & Cassia auriculata & Tangadi & Ceasalphinaceae & $\mathrm{P}$ & VO \\
\hline 02 & Lecuas & Leucas aspera & Thumbe & Lamiaceae & $\mathrm{N}$ & $\mathrm{VO}$ \\
\hline 03 & Tridax & Tridax procumbens & Sanna sevanthi & Asteraceae & $\mathrm{P}$ & $\mathrm{VO}$ \\
\hline 04 & $\begin{array}{l}\text { Congress } \\
\text { weed }\end{array}$ & Parthenium hysterophorus & Thurike gida & Asteraceae & $\mathrm{P}$ & $\mathrm{VO}$ \\
\hline 05 & Ageratum & Ageratum conyzoides & Ageratum & Asteraceae & $\mathrm{P}$ & $\mathrm{VO}$ \\
\hline 06 & Bidens & Bidens pilosa & Bidens gida & Asteraceae & NP & VO/MA \\
\hline 07 & Touch me not & Mimosa pudica & Muttidare muni & Fabaceae & $\mathrm{P}$ & VO \\
\hline 08 & Brahmi & Bacopa monniera & Brahmi & Scrgshulariaceae & $\mathrm{P}$ & $\mathrm{VO}$ \\
\hline 09 & $\begin{array}{l}\text { Mexican } \\
\text { poppy }\end{array}$ & Argemone Mexicana L. & Arasine umatti & Papavaraceae & $\mathrm{P}$ & $\mathrm{VO}$ \\
\hline 10 & Lantana & Lantana camera & Beligida & Verbanaceae & $\mathrm{P}$ & $\mathrm{VO}$ \\
\hline \multicolumn{7}{|c|}{$\begin{array}{ll}\text { VIII. } & \text { Trees } \\
\end{array}$} \\
\hline 01 & Eucalyptus & Eucalyptus globosa & Nilagiri & Myrtaceae & NP & $\mathrm{VO} / \mathrm{MA}$ \\
\hline 02 & Jamun & Syzgyium fruticosum & Nerale & Myrtaceae & NP & $\mathrm{VO} / \mathrm{MA}$ \\
\hline 03 & Jakaranda & Jacaranda acutifolia & Neeligulmohr & Bignoniaceae & NP & VO \\
\hline 04 & Tecoma & Tecoma stans & Obbattina gida & Bignoniaceae & NP & $\mathrm{VO} / \mathrm{MA}$ \\
\hline 05 & $\begin{array}{l}\text { Indian coral } \\
\text { tree }\end{array}$ & Erythrina indica & Halavana & Fabaceae & $\mathrm{P}$ & $\mathrm{VO}$ \\
\hline 06 & Babul & Acacia arabica W.II.d. & Karijali & Fabaceae & $\mathrm{P}$ & $\mathrm{VO}$ \\
\hline 07 & Gulmohr & Delonix regia Ref. & Kattikai & Fabaceae & NP & VO/MA \\
\hline 08 & Dhiancha & Sesbania grandiflora Pers. & Agase & Fabaceae & NP & $\mathrm{VO}$ \\
\hline 09 & Mesquite & Prosopis juliflora DC & Jali & Fabaceae & NP & VO/MA \\
\hline 10 & Pride of India & Lagerstromia indica L. & Telangana cheeni & Lythraceae & $\mathrm{P}$ & $\mathrm{VO}$ \\
\hline 11 & Camel foot & Bahunia purpurea & Basavana pada & Lythraceae & NP & $\mathrm{VO} / \mathrm{MA}$ \\
\hline
\end{tabular}




\begin{tabular}{|l|l|l|l|l|l|l|}
\hline 12 & Mehandi & Lawsonia inermis & Goranti & Lythraceae & NP & VO/MA \\
\hline 13 & Henna & Lawsonia inermos & Goranti & Lythraceae & NP & VO \\
\hline 14 & Copper pod & $\begin{array}{l}\text { Peltophorum petrocarpum } \\
\text { (DC \& Baker) }\end{array}$ & Bettadha hunese & Caesalpenaceae & NP & MA \\
\hline 15 & Pongamia & Pongamia glabra & Honge & Ceaesalpinaceae & N & VO/MA \\
\hline 16 & Cassia & Cassia javanica & Doddathangadhi & Ceaesalpinaceae & P & VO \\
\hline 17 & Sandal wood & Santalum album L. & Gandhada mara & Santalaceae & P & VO/MA \\
\hline 18 & Teak & Tectona grandis Linn. & Tega & Verbanaceae & NP & VO \\
\hline 19 & Nevuladi & Vitex peduncularis & Lakki & Verbanaceae & NP & VO \\
\hline 20 & $\begin{array}{l}\text { Seme } \\
\text { Nevuladi }\end{array}$ & Vitex trifolis & Lakki & Verbanaceae & NP & VO \\
\hline 21 & $\begin{array}{l}\text { Country } \\
\text { almond }\end{array}$ & Terminalia catappa & Dadami & Combretaceae & P & VO \\
\hline 22 & $\begin{array}{l}\text { Kodasege } \\
\text { gida }\end{array}$ & Holarrhena antidenterica & Kodasege gida & Apocyanaceae & NP & VO \\
\hline 23 & Ber & Zygypus nauritiana & Elachi & Rhamnaceae & NP & VO \\
\hline 24 & $\begin{array}{l}\text { White silk } \\
\text { cotton }\end{array}$ & Ceiba pentandra L. Gaertn & Doodhihatti & Bombaceae & P & VO \\
\hline 25 & Neem & Azadirichta indica Juss & Bevu & Meliaceae & N & VO/MA \\
\hline 26 & Silver oak & Gravellia robusta & Silver oak & Proteaceae & P & VO \\
\hline 27 & Rain tree & Samanea samon & Samane & Mimosaceae & P & MA \\
\hline
\end{tabular}

@ $\mathrm{P}=$ Source of pollen, $\mathrm{N}=$ Source of nectar, $\mathrm{VO}=$ Visual observation, $\mathrm{MA}=$ Microscopic analysis

Table.2 Family wise distribution of pasturage source of T. iridipennis

\begin{tabular}{|c|l|c|c|l|c|}
\hline Sl. No. & \multicolumn{1}{|c|}{ Family } & No. of plants & Sl. No. & Family & No. of plants \\
\hline 01 & Fabaceae & 14 & 24 & Solanaceae & 1 \\
\hline 02 & Verbanaceae & 5 & 25 & Caricaceae & 1 \\
\hline 03 & Lythraceae & 4 & 26 & Punicaceae & 1 \\
\hline 04 & Cucurbitaceae & 7 & 27 & Simaroubaceae & 1 \\
\hline 05 & Asteraceae & 9 & 28 & Eleocarpaceae & 1 \\
\hline 06 & Myrtaceae & 4 & 29 & Agavaceae & 1 \\
\hline 07 & Euphorbiaceae & 5 & 30 & Convolulaceae & 1 \\
\hline 08 & Ceasalpinaceae & 5 & 31 & Acanthaceae & 1 \\
\hline 09 & Vitaceae & 2 & 32 & Malvaceae & 1 \\
\hline 10 & Compositae & 2 & 33 & Rosaceae & 1 \\
\hline 11 & Umbelliferae & 4 & 34 & Balsaminae & 1 \\
\hline 12 & Apocyanaceae & 2 & 35 & Diascoreaceae & 1 \\
\hline 13 & Bignoniaceae & 2 & 36 & Onagraceae & 1 \\
\hline 14 & Poaceae & 4 & 37 & Scrgshulariaceae & 1 \\
\hline 15 & Lamiaceae & 3 & 38 & Papavaraceae & 1 \\
\hline 16 & Rutaceae & 3 & 39 & Meliaceae & 1 \\
\hline 17 & Brassicaceae & 1 & 40 & Proteaceae & 1 \\
\hline 18 & Anacardiaceae & 1 & 41 & Mimosaceae & 1 \\
\hline 19 & Moraceae & 2 & 42 & Santalaceae & 1 \\
\hline 20 & Arecaceae & 2 & 43 & Combretaceae & 2 \\
\hline 21 & Pedaliaceae & 1 & 44 & Rhamnaceae & 1 \\
\hline 22 & Moringaceae & 1 & 45 & Bombaceae & 1 \\
\hline 23 & Liliaceae & 1 & & & \\
\hline
\end{tabular}


Table.3 Plants identified for resin sources of Trigona iridipennis

\begin{tabular}{|l|l|l|l|l|l|}
\hline $\begin{array}{l}\text { Sl. } \\
\text { No. }\end{array}$ & $\begin{array}{l}\text { Common } \\
\text { name }\end{array}$ & Scientific name & Local name & Family & Source of resin \\
\hline 01 & Jack fruit & $\begin{array}{l}\text { Artocarpus heterophyllus } \\
\text { Lam. }\end{array}$ & Halasu & Moraceae & Cut branches, fruit stalk \\
\hline 02 & Drum stick & Moringa olifera (Lamk) & Nugge & Moringaceae & Stem, bark, cut branches \\
\hline 03 & Mango & Mangifera indica & Mavu & Anacardiaceae & Cut branches \\
\hline 04 & Perivinkle & Vinca rosea L. & Kasikanagalu & Apocyanaceae & Leaf lamina \\
\hline
\end{tabular}

Stingless bees required a large quantity of resin for nest building and was found to collect resin or latex from four species of plant which cut branches of mango, cut branches and fruit stalk of jack fruit, stem, bark and cut branches of drumstick and leaf lamina of perivinkle (Table 3).

Similar to pollen, resin was loaded in corbiculae and transported to back to the nest. In addition, these bees were found to collect fruit juice from cracked grape, mango and papaya. While foraging for resin they followed the method of group foraging. Such strategy with aggressiveness was also reported by Howard (1985).

Information on various pasturages sources will provide basis for evolving floral calendar of particular region and bee species.

\section{References}

Heard, T. A., 1999. The role of stingless bees in crop pollination. Ann. Rev. Entomol., 44:183-206.

Howard, J. J., 1985. Observations on resin collecting by six interacting species of stingless bees (Apidae: Meliponinae). $J$. Kans. Entomol. Soc., 58(2):337-345.

Ramalho, M., Guibu, L. S., Giannini, T. C., Kleinert, A. and Imperatriz, V. L., 1980. Characterization of some Southern Brazilian honey and bee plants through pollen analysis. J. Apic. Res., 30(2):8186.

Wille, A., 1983. Biology of the stingless bees. Ann. Rev. Entomol., 28:41-64.

\section{How to cite this article:}

Roopa, A.N., G. Eswarappa, Sanganna M. Sajjanar and Govi Gowda. 2017. Study on Identification of Pasturage Sources of Stingless Bee (Trigona iridipennis Smith.). Int.J.Curr.Microbiol.App.Sci. 6(11): 938-943. doi: https://doi.org/10.20546/ijcmas.2017.611.110 